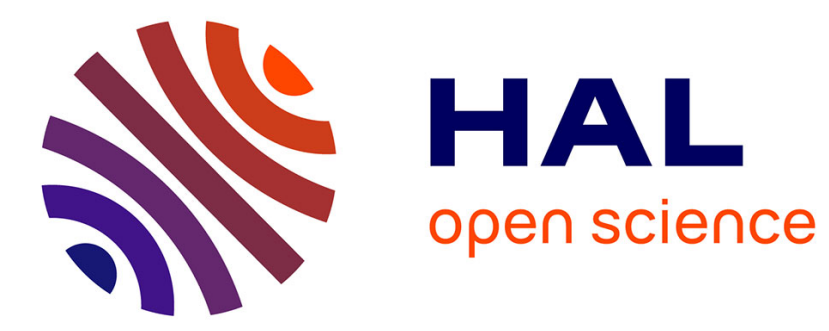

\title{
Quelques résultats sur certaines fonctions à lieu singulier de dimension 1
}

\author{
Daniel Barlet
}

\section{To cite this version:}

Daniel Barlet. Quelques résultats sur certaines fonctions à lieu singulier de dimension 1. 2006. hal00102131

\section{HAL Id: hal-00102131 \\ https://hal.science/hal-00102131}

Submitted on 29 Sep 2006

HAL is a multi-disciplinary open access archive for the deposit and dissemination of scientific research documents, whether they are published or not. The documents may come from teaching and research institutions in France or abroad, or from public or private research centers.
L'archive ouverte pluridisciplinaire HAL, est destinée au dépôt et à la diffusion de documents scientifiques de niveau recherche, publiés ou non, émanant des établissements d'enseignement et de recherche français ou étrangers, des laboratoires publics ou privés. 


\title{
Quelques résultats sur certaines fonctions à lieu singulier de dimension 1.
}

\author{
Daniel Barlet
}

$31 / 01 / 05$

\section{Abstract.}

This text is a survey on my recent work [B.04] on some holomorphic germs having a one dimensional singular locus. An analoguous of the Brieskorn module of an isolated singularity is defined and a finitness theorem is proved using Kashiwara's constructibility theorem. A bound for the(finite dimesional) torsion is also obtained.

Non existence of torsion is proved for curves (reduced or not) an this property is stable by "Thom-Sebastiani" adjunction of an isolated singularity. This provides a lot of examples in any dimension.

Key words. Hypersurface singularity, 1 dimensional singular locus, Brieskorn module, (a,b)-module, formal microlocal operators.

Classification. 32 S 05, 32 S 25, 32 S 40.

\section{Résumé.}

Nous nous proposons ici de donner un aperçu des résultats de notre article [B.04] dont le but est de développer, pour certains germes de fonctions holomorphes à l'origine de $\mathbb{C}^{n+1}$ dont le lieu singulier est de dimension 1, un analogue de la théorie de E. Brieskorn [Br. 70] pour les germes à singularité isolée. 


\section{$1 \quad$ Le complexe $\left(\operatorname{Ker} d f^{\bullet}, d^{\bullet}\right)$.}

Nous introduisons, de façon générale pour une fonction holomorphe $f$ sur une variété complexe $X$, le complexe $\left(\operatorname{Ker} d f^{\bullet}, d^{\bullet}\right)$ de faisceaux sur $Y:=f^{-1}(0)$ en posant :

$$
\operatorname{Ker} d f^{p}:=\operatorname{Ker}\left[\wedge d f:\left.\left.\Omega_{X}^{p}\right|_{Y} \rightarrow \Omega_{X}^{p+1}\right|_{Y}\right]
$$

et où la différentielle $d^{\bullet}$ est induite par la différentielle de de Rham.

Les faisceaux de cohomologie de ce complexe seront notés par $\mathcal{H}^{\bullet}$.

De façon générale ${ }^{1}$ ces faisceaux de cohomologie sont naturellement munis de deux opérations $a$ et $b$. La première est simplement donnée par la multiplication par $f$. La seconde est donnée par l'inverse de la connexion de Gauss-Manin, c'est à dire par $b=d f \wedge d^{-1}$. On a le résultat général suivant

\section{Proposition.}

Soit $f:\left(\mathbb{C}^{n+1}, 0\right) \rightarrow(\mathbb{C}, 0)$ un germe non nul de fonction holomorphe. Considérons pour $p \in[1, n+1]$ le $\mathbb{C}$-espace vectoriel

$$
\mathcal{H}_{0}^{p}:=\left((\operatorname{Ker} d f)^{p} \cap \operatorname{Ker} d\right)_{0} / d\left((\operatorname{Ker} d f)^{p-1}\right)_{0}
$$

muni des $\mathbb{C}$-endomorphismes $a$ et $b$ définis respectivement par la multiplication par $f$ et $d f \wedge d^{-1}$.

Alors $\mathcal{H}_{0}^{p}$ vérifie les conditions 0) 1) 2) et 3) de la définition d'un pré-(a,b)module donnée ci-dessous.

\section{Définition.}

Soit E un espace vectoriel complexe muni d'endomorphismes $a$ et $b$. On pose :

$$
B(E)=\bigcup_{m \in \mathbb{N}} \operatorname{Kerb}^{m} \quad \text { et } \quad A(E)=\left\{x \in E / \mathbb{C}[b] . x \subset \bigcup_{m \in \mathbb{N}} \operatorname{Ker}^{m}\right\}
$$

On dira que $E$ est un pré-(a,b)-module lorsque les conditions suivantes sont vérifiées

\footnotetext{
${ }^{1}$ c'est à dire sans hypothèse sur $f$.
} 
- 0) $a . b-b . a=b^{2}$.

- 1) Pour tout $\lambda \in \mathbb{C}^{*}, b-\lambda$ est bijectif dans $E$.

- 2) $\exists N \in \mathbb{N} / a^{N} \cdot A(E)=0$ et on a $B(E) \subset A(E)$.

- 3) $\bigcap_{m \in \mathbb{N}} b^{m}(E) \subset A(E)$.

- 4) Le noyau et le conoyau de $b$ sont de dimensions finies sur $\mathbb{C}$.

On dira que $E$ est sans torsion ${ }^{2}$ si on a de plus $\operatorname{Ker} b=0$, ce qui équivaut à $B(E)=0$ et donc à l'absence de $b$-torsion. Mais ceci implique aussi $A(E)=0$ (voir [B.04] Lemme (1.3)). Dans le cas considéré dans la proposition ci-dessus le théorème de positivité de B. Malgrange [M. 74] donne alors l'absence de $a$-torsion ${ }^{3}$ (voir aussi [B.S.04]).

A tout pré-(a,b)-module $E$ on associe un (a,b)-module, c'est à dire un module libre de type fini sur l'anneau $\mathbb{C}[[b]]$ muni d'un endomorphisme $\mathbb{C}$-linéaire $a$ qui est continu pour la topologie $b$-adique et vérifie

$$
a b-b a=b^{2} .
$$

Nous le noterons par $\mathcal{L}(E)$ et c'est le complété $b$-adique du quotient $E / B(E)$. On remarquera que grace à la relation 3 ) (et à l'égalité entre $B(E)$ et $A(E))$ on a $b$-séparation de ce quotient. Donc $E / B(E)$ s'injecte toujours dans $\mathcal{L}(E)$. Quand $E$ est sans torsion, on en déduit que la dimension complexe de $E / b E$ est le rang du (a,b)-module $\mathcal{L}(E)$. En général ce rang est donné par $\operatorname{dim} E / b E-\delta$ où $\delta:=\operatorname{dim} \operatorname{Ker} b$.

Les $(\mathrm{a}, \mathrm{b})$-modules que l'on associe à des singularités sont loin d'être les plus "généraux"; en particulier ils sont réguliers, propriété qui reflète la regularité de la connexion de Gauss-Manin. Pour plus de détails la-dessus consulter par exemple [B. 93], [B.04] ou [B.S.04]).

\footnotetext{
${ }^{2}$ même en l'absence de la condition 4).

${ }^{3}$ ce qui est plus fort, en général, que la nullité de $A(E)$.
} 


\section{L'hypothèse $(H)$.}

Soit $\tilde{f}:\left(\mathbb{C}^{n+1}, 0\right) \longrightarrow(\mathbb{C}, 0)$ un germe non constant de fonction holomorphe et soit $f: X \longrightarrow D$ un représentant de Milnor de $\tilde{f}$. Nous ferons les hypothèses suivantes (auxquelles nous nous référons sous le nom de "l'hypothèse $(\mathrm{H})$ ")

$H$ a) Le lieu singulier $S:=\left\{x \in X / d f_{x}=0\right\}$ est une courbe contenue dans $Y:=f^{-1}(0)$, dont chaque composante irréductible contient l'origine et est non singulière en dehors de 0 .

$H$ b) En chaque point $x$ de $S-\{0\}$ il existe un germe en $x$ de champ de vecteur holomorphe $V_{x}$, non nul en $x$, tel que $V_{x} \cdot f \equiv 0$.

L'hypothèse $H \quad b$ ) est assez restritive puisqu'elle implique que le long de chaque composante connexe de $S-\{0\}$ la singularité $\{f=0\}$ est une déformation localement analytiquement triviale de la singularité hyperplane transverse (qui est une singularité isolée de $\mathbb{C}^{n}$ ).

Cependant il est facile de voir que cette hypothèse est toujours vérifiée pour $n=1$ (courbes planes réduites ou non ) et qu'il y a beaucoup d'exemples en dimensions supérieures, comme le montrera le théorème 11.

\section{Proposition.}

Sous l'hypothèse $(\mathrm{H})$ on a les propriétés suivantes :

- 1) Les faisceaux de cohomologie $\mathcal{H}^{p}$ du complexe $\left(\operatorname{Ker} d f^{\bullet}, d^{\bullet}\right)$ sont nuls pour $p \neq 1, n, n+1$.

- 2) Pour $n \geq 2$ le faisceau $\mathcal{H}^{1} \simeq K e r d f^{1}$ est constant sur $Y$ de fibre isomorphe à $E_{1}=\Omega_{\mathbb{C}, 0}^{1}$ muni des opérations $a$ et $b$ "naturelles" de multiplication par $z$ et de "primitive sans constante" .

- 3) Pour $n \geq 2$ le faisceau $\mathcal{H}^{n}$ a son support contenu dans $S$. Il est localement constant sur $S^{*}:=S \backslash\{0\}$. Sa fibre en un point d'une composante connexe $S_{j}^{*}$ de $S^{*}$ est le module de Brieskorn de la singularité obtenue par section hyperplane transverse. De plus le faisceau $\mathcal{H}^{n}$ n'a pas de torsion (même à l'origine). 
- 4) Le faisceau $\mathcal{H}^{n+1}$ est supporté par l'origine.

- 5) Pour $n=1$ on a une suite exacte de faisceaux sur $Y$, compatible aux opérations $a$ et $b$ :

$$
0 \rightarrow E_{1} \otimes \underline{\mathbb{C}}_{Y} \rightarrow \mathcal{H}^{1} \rightarrow \tilde{\mathcal{H}}^{1} \rightarrow 0
$$

où le faisceau $\tilde{\mathcal{H}}^{1}$ a son support dans $S$ et induit sur $S^{*}$ un système local évident à décrire ${ }^{4}$.

Introduisons maintenant deux ingrédients importants dans cette situation.

\section{L'idéal $\widehat{J}(f)$.}

Notons par $J(f)$ l'idéal jacobien de $f$ et soit $i: X \backslash\{0\} \hookrightarrow X$ l'inclusion, où $X$ désigne ici une boule de Milnor pour $f$ à l'origine. Définissons alors l'idéal (cohérent) $\widehat{J}(f):=i_{*} i^{*}(J(f))$. Donc un germe $g$ de fonction holomorphe à l'origine sera dans $\widehat{J}(f)_{0}$ si et seulement s' il induit une section de $J(f)$ en dehors de $0^{5}$. Le faisceau cohérent $\widehat{J}(f) / J(f)$ est à support l'origine. C'est donc un espace vectoriel de dimension finie. Nous poserons

$$
\mu(f):=\operatorname{dim} \widehat{J}(f) / J(f)
$$

ce qui définit l'analogue du nombre de Milnor pour le cas d'une fonction à singularité isolée ${ }^{6}$.

\section{$7 \quad$ Le $\mathcal{D}_{X}-$ module $\mathcal{M}$.}

Définissons maintenant l'idéal à gauche $\mathcal{I}$ de $\mathcal{D}_{X}$ comme l'déal engendré par $\widehat{J}(f)$ et par l'annulateur de $f$ dans le faisceau $\Theta_{X}$ des champs de vecteurs holomorphes sur $X$ :

$$
A n n(f)=\left\{V \in \Theta_{X} / V . f \equiv 0\right\} .
$$

Posons alors $\mathcal{M}:=\mathcal{D}_{X} / \mathcal{I}$.

\footnotetext{
${ }^{4}$ la singularité transverse se réduit à la multiplicité transverse dans ce cas.

${ }^{5}$ nous supposerons toujours $n \geq 1$ ce qui permet d'utiliser Hartogs.

${ }^{6}$ dans ce cas $\widehat{J}(f)=\mathcal{O}_{X}$.
} 
Il est clair que l'hypothèse $(\mathrm{H})$ implique immédiatement que $\mathcal{M}$ est un $\mathcal{D}_{X}-$ module holonome de support contenu dans $S$. D'après le théorème de constructibilité de M. Kashiwara [K. 75] son complexe de de Rham est à cohomologie constructible. En particulier, le faisceau $D R^{n+1}(\mathcal{M})$ est concentré à l'origine et se réduit à un espace vectoriel de dimension finie. Nous poserons

$$
\nu(f):=\operatorname{dim} D R^{n+1}(\mathcal{M}) .
$$

On remarquera que pour $f$ à singularité isolée à l'origine on a $\mathcal{M}=0$ puisque $\widehat{J}(f)=\mathcal{O}_{X}$. On a donc $\nu(f)=0$ dans ce cas.

\section{Théorème.}

Sous l'hypothèse $(\mathrm{H})$ l'espace vectoriel $E^{\prime}:=\mathcal{H}_{0}^{n+1} \simeq H_{\{0\}}^{0}\left(Y, \mathcal{H}^{n+1}\right)$ est un pré-(a,b)-module de rang $r$ vérifiant

$$
r=\mu(f)+\nu(f)-\delta \leq \mu(f)+\nu(f)
$$

où $\delta$ est la dimension de $\operatorname{Ker} b$ dans $E^{\prime}$. De plus on a la majoration :

$$
\delta \leq \operatorname{dim} H_{\{0\}}^{1}\left(S, \mathcal{H}^{n} / \text { b. } \mathcal{H}^{n}\right) .
$$

On a $\delta=0$ et donc l'égalité $r=\mu(f)+\nu(f)$ sous la condition (P) décrite ci-dessous.

On remarquera que comme $\mathcal{H}^{n} / b . \mathcal{H}^{n}$ est un système local d'espaces vectoriels de dimensions finies sur $S^{*}$, on a bien une majoration finie de $\delta$. Par ailleurs ce majorant est en pratique assez facile à estimer.

\section{La condition $(\mathrm{P})$.}

Comme le fait E. Brieskorn dans le cas d'une fonction à singularité isolée à l'origine, il est intéressant d'introduire également le faisceau

$$
\mathcal{E}^{\prime \prime}:=\Omega_{X}^{n+1} / d f \wedge d \Omega_{X}^{n-1}
$$

en plus du faisceau $\mathcal{E}^{\prime} \equiv \mathcal{H}^{n+1}$. Il est également muni d'opérations $a$ et $b$ naturelles vérifiant la relation de commutation $a b-b a=b^{2}$. On a alors une surjection naturelle compatible à $a$ et $b$

$$
j: \mathcal{E}^{\prime \prime} \rightarrow \mathcal{E}^{\prime}
$$


de noyau $d f \wedge \Omega_{X}^{n} / d f \wedge d \Omega_{X}^{n-1}$. Mais le faisceau $\mathcal{E}^{\prime \prime}$ n'est pas, en général, supporté par l'origine sous l'hypothèse $(\mathrm{H})^{7}$.

Posons :

$$
E^{\prime}:=H_{\{0\}}^{0}\left(X, \mathcal{E}^{\prime}\right) \quad \text { et } \quad E^{\prime \prime}:=H_{\{0\}}^{0}\left(X, \mathcal{E}^{\prime \prime}\right) .
$$

La suite exacte de cohomologie à support l'origine déduite du morphisme $j$ donne une suite exacte (a,b)-linéaire

$$
0 \rightarrow H_{\{0\}}^{0}(X, \operatorname{Ker} j) \rightarrow E^{\prime \prime} \stackrel{\hat{j}}{\rightarrow} E^{\prime} \rightarrow \cdots
$$

On a également une suite exacte de faisceaux

$$
0 \rightarrow \mathcal{E}^{\prime} \stackrel{\tilde{b}}{\rightarrow} \mathcal{E}^{\prime \prime} \rightarrow \Omega_{X}^{n+1} / d f \wedge \Omega_{X}^{n} \rightarrow 0
$$

où le morphisme $\tilde{b}$ est donné par $d f \wedge d^{-1}$. Comme le faisceau $\mathcal{E}^{\prime}$ est à support l'origine, on en déduit la suite exacte

$$
0 \rightarrow E^{\prime} \stackrel{\tilde{b}}{\rightarrow} E^{\prime \prime} \rightarrow H_{\{0\}}^{0}\left(X, \Omega_{X}^{n+1} / d f \wedge \Omega_{X}^{n}\right) \rightarrow 0
$$

et l'application $b: E^{\prime} \rightarrow E^{\prime}$ est la composée $\hat{j} \circ \tilde{b}$. Comme $\tilde{b}$ est injective, l'injectivité de $\hat{j}$ implique celle de $b$ dans $E^{\prime}$. La condition suivante est necessaire et suffisante pour que l'application $\hat{j}$ soit injective :

$$
d\left(\operatorname{Ker} d f^{n}\right) \cap \widehat{J}(f) . \Omega_{X}^{n+1} \subset d f \wedge d \Omega_{X}^{n-1}
$$

alors qu'une condition nécessaire et suffisante pour avoir l'injectivité de $b: E^{\prime} \rightarrow E^{\prime}$ est donnée par l'inclusion :

$$
d\left(\operatorname{Ker} d f^{n}\right) \cap\left(d f \wedge \Omega_{X}^{n}\right) \subset d f \wedge d \Omega_{X}^{n-1} .
$$

Bien sur, la condition $(\mathrm{P})$ implique la condition $\left(\mathrm{P}^{\prime}\right)$. Mais il est bon de préciser, qu'à ce jour, je ne connais pas d'exemple de germe vérifiant l'hypothèse $(\mathrm{H})$ et ne vérifiant pas la condition $(\mathrm{P})$. Malheureusement, je n'ai pas non plus de preuve que l'hypothèse $(\mathrm{H})$ implique toujours la condition $(\mathrm{P})$. C'est cependant vrai (et non trivial) pour $n=1$ :

\footnotetext{
${ }^{7}$ comme le montre la suite exacte $(\mu)$ ci-dessous.
} 


\section{Théorème.}

Pour $n=1$, c'est à dire pour les germes à l'origine de $\mathbb{C}^{2}$, réduits ou non, la condition $(\mathrm{P})$ est toujours vérifiée.

La preuve de ce résultat utilise la récurrence tordue suivante qui permet de tenir compte des multiplicités d'annulation d'une fonction sur les différentes composantes irréductibles de $Y^{8}$.

\subsection{Lemme (récurrence tordue).}

Soient $\phi_{1} \cdots \phi_{k}$ des fonctions strictement croissantes

$$
\phi_{j}:\left[0, p_{j}-1\right] \longrightarrow[0,1]
$$

vérifiant

$$
\phi_{j}\left(p_{j}-1\right)=1, \forall j \in[1, k] .
$$

Considérons des propositions $A\left(\sigma_{1}, \cdots, \sigma_{k}\right)$ indéxées par les entiers

$$
\sigma_{j} \in\left[0, p_{j}-1\right] \text {. }
$$

On suppose

- 1) $A(0, \cdots, 0)$ est vraie .

- 2) l'implication $A\left(\sigma_{1}, \cdots, \sigma_{k}\right) \Rightarrow A\left(\sigma_{1}, \cdots, \sigma_{j+1}, \cdots, \sigma_{k}\right)$ est vraie si les deux conditions suivantes sont satisfaites

$$
\begin{aligned}
& \text { a) } \sigma_{j} \leq p_{j}-2 \\
& \text { b) } \phi_{j}\left(\sigma_{j}\right)=\operatorname{In} f_{l \in[1, k]}\left\{\phi_{l}\left(\sigma_{l}\right)\right\}
\end{aligned}
$$

Alors la proposition $A\left(p_{1}-1, \cdots, p_{k}-1\right)$ est vraie .

On prendra garde que nous n'affirmons pas ici que $A\left(\sigma_{1}, \cdots, \sigma_{k}\right)$ est vraie pour toutes les valeurs de $\left(\sigma_{1}, \cdots, \sigma_{k}\right)$.

Le résultat suivant fournit, à partir de ce théorème, une bonne quantité d'exemples de fonctions vérifiant l'hypothèse $(\mathrm{H})$ pour lesquelles la condition (P) est vraie :

\footnotetext{
${ }^{8}$ rappelons que l'on est ici dans $\mathbb{C}^{2}$ et donc que $Y$ est une courbe et que $S$ est la réunion des composantes irréductibles de $Y$ de multiplicité $\geq 2$.
} 


\section{Theorème.}

Soit $f$ un germe de fonction à singularité isolée à l'origine de $\mathbb{C}^{n+1}$ et soit $g$ un germe de fonction holomorphe à l'origine de $\mathbb{C}^{p+1}$ vérifiant l'hypothèse $(\mathrm{H})$. Alors le germe de fonction holomorphe $F$ à l'origine de $\mathbb{C}^{n+p+2}$ défini par

$$
F(x, y):=f(x)+g(y) \quad \text { pour } \quad(x, y) \in \mathbb{C}^{n+1} \times \mathbb{C}^{p+1}
$$

vérifie $(\mathrm{H})$. De plus, le (a,b)-module associé à $F$ à l'origine est le produit tensoriel $^{9}$ des $(\mathrm{a}, \mathrm{b})$-modules associés à l'origine à $f$ et $g$ respectivement. $\mathrm{Si}$, de plus, le germe $g$ vérifie la condition $(\mathrm{P})$, il en est de même pour $F$.

Dans la situation du théorème ci-dessus, on peut également décrire le faisceau $\mathcal{H}^{n+p+1}$ associé à $F^{10}$ à partir du module de Brieskorn de $f$ et du faisceau $\mathcal{H}^{n}$ associé à $g$.

\section{Produits tensoriels de $(a, b)$-modules.}

Nous allons nous contenter ici de définir le produit tensoriel de deux (a,b)modules, ce qui permet de comprendre le résultat à la "Thom-Sébastiani" énoncé ci-dessus.

\section{Définition.}

Etant donné deux (a,b)-modules $E$ et $F$ nous poserons

$$
E \otimes_{a, b} F:=E \otimes_{\mathbb{C}[[b]]} F
$$

comme $\mathbb{C}[[b]]$-module, et nous définirons l'endomorphisme $\mathbb{C}$-linéaire $a$ en posant

$$
a:=a_{E} \otimes 1_{F}+1_{E} \otimes a_{F} .
$$

On vérifie alors immédiatement la relation de commutation $a b-b a=b^{2}$ sur $E \otimes_{a, b} F$. Comme un (a,b)-module est complètement déterminé par la donnée d'une $\mathbb{C}[[b]]$-base ainsi que l'action de $a$ sur cette base (grace à la relation

\footnotetext{
${ }^{9}$ au sens des (a,b)-modules ; voir ci-dessous.

${ }^{10}$ ou plus exactement le faisceau de $(\mathrm{a}, \mathrm{b})$-module qui lui est associé après complétion formelle.
} 
de commutation et à la continuité de $a$ pour la topologie $b$-adique) il est facile de décrire en ces termes le produit tensoriel de deux (a,b)-modules donnés de cette façon.

En fait dans [B.04] on définit également la notion de produit tensoriel de deux pré-(a,b)-modules et on montre, sous une condition très large ${ }^{11}$, que l'on obtient à nouveau un pré-(a,b)-module. On montre alors que le (a,b)module associé au produit tensoriel est le produit tensoriel des (a,b)-modules associés.

\section{Un exemple.}

Considérons le polynôme homogène à deux variables ${ }^{12}$

$$
f(X, Y)=X^{3}\left(X^{3}+Y^{3}\right) .
$$

Il est facile de voir que $\operatorname{Ker} d f^{1} / \mathcal{O} . d f$ est engendré par la 1 -forme associé au champ de vecteur

$$
V=X Y^{2} \frac{\partial}{\partial X}-\left(2 X^{3}+Y^{3}\right) \frac{\partial}{\partial Y}
$$

annulant $f$ et de divergence $\operatorname{div} V=-2 Y^{2}$. Posons $\tilde{V}=V-\operatorname{div}(V)$.

On a $J(f)=X^{2} \cdot\left(2 X^{3}+Y^{3}, X Y^{2}\right), \widehat{J(f)}=\left(X^{2}\right)$ et $\operatorname{dim}_{\mathbb{C}} \widehat{J(f)} / J(f)=\mu(f)=9$.

Par ailleurs, en explicitant $D R^{2}(\mathcal{M})$ on trouve :

$$
\nu(f)=\operatorname{dim} \mathcal{O}_{X} /(\widehat{J}(f))+\tilde{V}\left(\mathcal{O}_{X}\right) .
$$

Pour calculer $\operatorname{dim}_{\mathbb{C}}\left(\mathbb{C}\{X, Y\} /\left(X^{2}\right)+\widetilde{V}(\mathbb{C}\{X, Y\})\right)$ on regarde l'identité

$$
\widetilde{V}\left(X^{p} Y^{q}\right)=(p-q-2) X^{p} Y^{q+2}-2 q X^{p+3} Y^{q-1} ;
$$

on peut donc réduire $X^{a} Y^{b}$ à $X^{a+3} Y^{b-3}$ pourvu que $a \neq b$ et $b \geq 2$.

\footnotetext{
${ }^{11}$ l'hypothèse "locale", à savoir l'existence d'un entier $l$ tel que $a^{l} . E \subset b . E$; elle est toujours satisfaite dans les cas que nous considérons.

${ }^{12}$ pour $n=1$ l'hypothèse $(\mathrm{H})$ est toujours vérifiée, ainsi que la condition $(\mathrm{P})$ comme nous l'avons vu plus haut.
} 
Si $a=b \geq 2$ on est dans $\left(X^{2}\right)$. Il reste donc $1, X, Y$ et $X^{p} Y$ pour $p<2$. Donc $1, X, Y, X Y$ donne une base et $\nu(f)=4$.

Alors $\mathcal{L}\left(E^{\prime}\right)^{13}$ est un $(a, b)$-module de rang 13 . Une base de $E^{\prime} / b E^{\prime}$ est donnée par

$$
1, X, Y, X Y, X^{2}, X^{3}, X^{4}, X^{5}, X^{2} Y, X^{3} Y, X^{4} Y, X^{5} Y, X^{2} Y^{2} .
$$

Comme on a

$$
b\left(X^{p} Y^{q}\right) d X \wedge d Y=d f \wedge \frac{X^{p+1} Y^{q}}{p+1} d Y=-d f \wedge \frac{X^{p} Y^{q+1}}{q+1} d X,
$$

on en déduit que

$$
a\left[X^{p} Y^{q}\right]=\frac{p+q+2}{6} \cdot b\left[X^{p} Y^{q}\right]
$$

Pour calculer le $(\mathrm{a}, \mathrm{b})$-module associé à l'origine au polynôme à trois variables $F(X, Y, Z)=X^{3}\left(X^{3}+Y^{3}\right)+Z^{2}$ il nous suffit donc de calculer le (a,b)-module de Brieskorn associé à la fonction $Z \rightarrow Z^{2}$ et de faire le produit tensoriel avec celui associé à $X^{3}\left(X^{3}+Y^{3}\right)$ à l'origine. Le (a,b)-module de Brieskorn associé à $Z \rightarrow Z^{2}$ est de rang 1 et engendré par un générateur $e$ vérifiant a.e $=\frac{1}{2} . b . e$. Le produit tensoriel est donc de rang 13 et engendré par les $X^{p} Y^{q} \otimes e$ mais avec

$$
a\left[X^{p} Y^{q} \otimes e\right]=\frac{p+q+5}{6} b\left[X^{p} Y^{q} \otimes e\right] .
$$

\footnotetext{
${ }^{13}$ Le complété $b$-adique de $E^{\prime}$, puisque $\operatorname{Ker} b=0$ et donc $B(E)=A(E)=0$.
} 


\section{Références.}

- [B.93] Barlet, D. Théorie des $(a, b)$-modules I, in Complex Analysis and Geometry, Plenum Press, (1993), p 1-43.

- [B.04] Barlet, D. Sur certaines singularités non isolées d'hypersurfaces I, preprint Institut E. Cartan 2004/ $n^{0}$ 03, 47 pages.

- [Br. 70] Brieskorn, E. Die Monodromie der isolierten Singularitäten von Hyperflächen, Manuscripta Math. 2 (1970), p.103-161

- [B.S. 04] Barlet, D. and Saito, M. Brieskorn modules and Gauss-Manin systems for non isolated hypersurface singularities, preprint Institut E. Cartan 2004/ $n^{0}$ 54, 16 pages.

- [K. 75] Kashiwara, M. On the maximally over determined systems of differential equations, Publ. R.I.M.S. 10 (1975), p. 563-579.

- [M. 74] Malgrange,B. Intégrale asymptotique et monodromie, Ann. Sc. Ec. Norm. Sup. 7 (1974), p. 405-430.

Daniel Barlet ,

Université Henri Poincaré (Nancy I ) et Institut Universitaire de France, Institut E.Cartan UHP/CNRS/INRIA, UMR 7502 ,

Faculté des Sciences et Techniques, B.P. 239

54506 Vandoeuvre-les-Nancy Cedex , France.

e-mail : barlet@iecn.u-nancy.fr 